\title{
Effects of electron-electron interactions in suspended graphene
}

\section{Popovici*}

Institut für Theoretische Physik, Justus-Liebig-Universität Giessen Heinrich-Buff-Ring 16, 35392 Giessen, Germany

E-mail: carina.popovici@theo.physik.uni-giessen.de

\section{S. Fischer}

Institut für Theoretische Physik, Justus-Liebig-Universität Giessen Heinrich-Buff-Ring 16, 35392 Giessen, Germany

\section{L. von Smekal}

Theoriezentrum, Institut für Kernphysik, Technische Universität Darmstadt,

Schlossgartenstraße 2, 64289 Darmstadt, Germany

\begin{abstract}
We investigate the problem of dynamical gap generation in suspended graphene by long-range Coulomb interactions at strong coupling with Dyson-Schwinger equations. Including renormalization effects on the Fermi velocity we obtain a critical coupling constant $\alpha_{c}=2.85$ which is larger than the bare coupling $\alpha_{0}=2.19$ of suspended graphene. This suggests that at low energies the running of the Fermi velocity prevents the emergence of a gapped phase. Our calculation is thus in agreement with the experimental observation that suspended graphene remains in the semimetal phase.
\end{abstract}

Xth Quark Confinement and the Hadron Spectrum

8-12 October 2012

TUM Campus Garching, Munich, Germany

\footnotetext{
* Speaker.
} 


\section{Introduction}

Due to its unusual electronic properties, graphene, a monolayer of carbon atoms on a twodimensional honeycomb lattice [1], has been intensely studied both theoretically and experimentally over the last years (see, for example, Refs. [2, 3, 4] for overviews). In particular, much work has been devoted to understanding the effects of electron-electron interactions, both in the continuum, mainly via Dyson-Schwinger and renormalization group equations $[5,6,7,8,9,10,11,12$, $13,14,15]$, and on the lattice $[16,17,18,19,20]$. The majority of the theoretical efforts were thereby focussed on the question whether the long-range Coulomb interactions might trigger the dynamical generation of a gap in graphene at strong coupling similar to chiral symmetry breaking in QCD. This possibility is motivated by the potentially large value of the effective coupling $\alpha=e^{2} / \varepsilon \hbar v_{F}$, where $v_{F} \sim 10^{6} \mathrm{~m} / \mathrm{s} \approx c / 300$ is the Fermi velocity of graphene on a substrate with dielectric constant $\varepsilon$. If the Fermi velocity of suspended graphene with $\varepsilon=1$ is of the same order, this could lead to the formation of a condensate corresponding to a transition into an insulating phase above some critical coupling $\alpha_{c}$.

In a series of theoretical works, various authors have calculated the value of the critical coupling that would correspond to the semimetal-insulator transition: Gamayun et al. found $\alpha_{c}=0.92$ by solving a simplified gap equation in which radiative corrections to the Fermi velocity are neglected [10]; in [12], an approximation for the photon polarization has been used, leading to the critical value $\alpha_{c}=1.13$; renormalization group calculations at two loops yield $\alpha_{c}=0.833$ [21]; on the lattice, calculations performed using a 'standard' square lattice found the value $\alpha_{c}=1.08 \pm 0.05$ [17], whereas simulations carried out directly on a hexagonal (physical) lattice yield $\alpha_{c}=0.9 \pm 0.2$ [19]. All these values lie below the bare coupling constant of suspended graphene, $\alpha_{0}=2.19$.

On the other hand, the experimentally measured conductivity shows that suspended graphene is not in the insulating phase [4, 22]. It is observed, however, that the strong Coulomb interactions induce a charge-carrier density dependent renormalization of the Fermi velocity corresponding to a reshaping of the Dirac cones. This renormalization peaks at half-filling where it leads to an increase by about a factor of three in the Fermi velocity and thus a corresponding decrease in the renormalized effective coupling. A strong renormalization of $v_{f}$ was predicted already in an early perturbative RG study [5] where it was concluded that the Coulomb interactions would in fact induce a logarithmic increase in the Fermi velocity without bound until retardation effects eventually become important and the instantaneous Coulomb approximation breaks down.

While lattice simulations can yield reliable results in strongly coupled theories, extrapolations to the infinite volume and chiral limits are often a numerical challenge. This is also very much true for the simulations of the electronic properties of graphene. Therefore, functional methods in which these limits are accessed more easily can provide important alternative nonperturbative formulations. Moreover, the inevitable approximations in these formulations can be validated from simulations with sufficiently large masses in finite volumes. Here we employ the Dyson-Schwinger equations. Within this approach, substantial progress has been made over the past decades, both in in QCD and $\mathrm{QED}_{3}$ (see, for example, Refs. [23, 24, 25, 26, 27] and references therein). Extending the study of Ref. [10], we first calculate the running Fermi velocity in the bare vertex approximation and with a one-loop (frequency dependent) photon polarization. In a second step, we solve the gap equation at the critical point by explicitly including the dressing function corresponding to 
this running Fermi velocity. For the critical coupling of the semimetal-insulator transition we then obtain $\alpha_{c}=2.85$ [28] which is larger than the bare coupling of suspended graphene $\alpha_{0}=2.19$, indicating the persistence of the semimetal phase. Our results thus agree with the experimental findings [22]. Finally, in order to compare our results with the lattice simulations which must include a sufficiently large mass term in a finite volume, we have also investigated the dependence of the corresponding pseudocritical coupling on such a fermion mass term which acts as an explicitly symmetry breaking external field as the current quark masses do in QCD.

\section{Running Fermi velocity and critical point analysis}

We start by briefly sketching the details of the continuum model of graphene employed in this work. At the level of the Lagrangian, including the Coulomb interaction corresponds to accommodating the boson field that propagates in $(3+1)$ dimensions into the $(2+1)$ dimensional Lagrangian of the massless Dirac fermions. This is done via dimensional reduction, which gives rise to a so-called reduced gauge theory (we refer to Refs. $[9,10,28]$ for a complete description and derivation). The dimensionally reduced action of 2D Dirac fermions interacting through a three-dimensional gauge field is given by

$$
\begin{aligned}
\mathscr{S}= & \int d t d^{2} \vec{r} \bar{\psi}(t, \vec{r})\left[\mathrm{i} \gamma^{0} \partial_{t}-\mathrm{i} v_{F} \gamma^{i} \partial_{i}\right] \psi(t, \vec{r}) \\
& -\frac{e^{2}}{2 \varepsilon} \int d t d t^{\prime} d^{2} \vec{r} d^{2} \vec{r}^{\prime} \delta\left(t-t^{\prime}\right) \bar{\psi}(t, \vec{r}) \gamma^{0} \psi(t, \vec{r}) \frac{1}{\left|\vec{r}-\vec{r}^{\prime}\right|} \bar{\psi}\left(t^{\prime}, \vec{r}^{\prime}\right) \gamma^{0} \psi\left(t^{\prime}, \vec{r}^{\prime}\right) .
\end{aligned}
$$

Here, the spatial index is $i=1,2$, and $s=1, \ldots, N_{f}$ is a flavor quantum number with $N_{f}=2$ for monolayer graphene referring to the physical spin degree of freedom. $\psi(\bar{\psi})$ denotes the fermion (conjugate) field, $v_{F}$ is the Fermi velocity, and $\varepsilon$ is the dielectric constant of the medium (for suspended graphene $\varepsilon=1$, whereas on top of a substrate one uses $\varepsilon=(1+\kappa) / 2$ typically with $\kappa \approx 4$ for $\mathrm{SiO}_{2}$ or $\kappa \approx 10$ for $\mathrm{SiC}$ ).

From the action in Eq. (2.1) one obtains the fermion Dyson-Schwinger (gap) equation via standard functional derivation. In Minkowski space we have

$$
S^{-1}\left(p_{0}, \vec{p}\right)=S_{0}^{-1}\left(p_{0}, \vec{p}\right)-\mathrm{i} e \gamma^{0} \int \frac{d^{3} k}{(2 \pi)^{3}} S\left(k_{0}, \vec{k}\right) \Gamma(k,-p) D\left(p_{0}-k_{0}, \vec{p}-\vec{k}\right),
$$

where $\Gamma(k,-p)$ is the fully dressed fermion-photon vertex and the fermion propagator is given by (without wavefunction renormalization, details will be provided elsewhere [28]):

$$
S(p)=\frac{\gamma^{0} p_{0}-v_{F} A(p) \gamma^{i} p_{i}-\Delta(p)}{p_{0}^{2}-v_{F}^{2} A^{2}(p) \vec{p}^{2}-\Delta^{2}(p)}
$$

where $A$ is the Fermi velocity dressing function, and $\Delta$ is the fermion mass function. The Coulomb propagator in the random-phase approximation (RPA),

$$
D\left(q_{0}, \vec{q}\right)=\frac{2 \pi}{|\vec{q}|+\Pi\left(q_{0}, \vec{q}\right)},
$$

includes polarization effects with the one-loop polarization function [5],

$$
\Pi\left(q_{0}, \vec{q}\right)=\frac{\pi e^{2} N_{f}}{4 \varepsilon} \frac{\vec{q}^{2}}{\sqrt{\hbar^{2} v_{F}^{2} \vec{q}^{2}-q_{0}^{2}}} .
$$




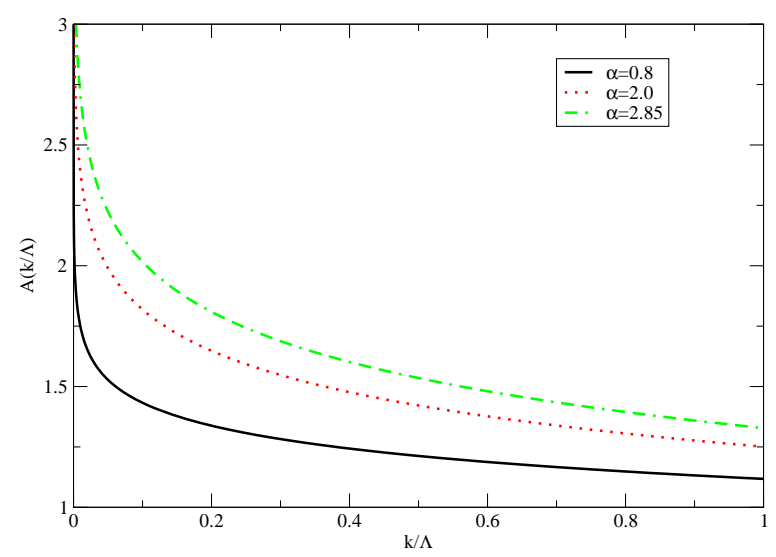

Figure 1: The Fermi velocity dressing function $A$ in GWA with RPA Coulomb interactions, $\Lambda \sim \hbar / a$, $N_{f}=2$ for monolayer graphene, and $\alpha=0.8,2.0$ and 2.85 to represent the presence of a substrate, suspended graphene, and criticality for the semimetal-insulator transition, respectively, from bottom to top.

Following the usual procedure, we recast the Dyson-Schwinger equation Eq. (2.2) into a set of coupled integral equations for the dressing functions $A(p)$ and $\Delta(p)$. Neglecting a possible weak energy dependence in $A$ and $\Delta$, and in a bare vertex truncation $\Gamma \rightarrow \Gamma^{(0)}=e \gamma^{0}$, one obtains [10]:

$$
\begin{aligned}
& A(\vec{p})=1+\frac{e^{2}}{\varepsilon} \int_{-\infty}^{\infty} \frac{d \omega}{2 \pi} \int \frac{d^{2} \vec{k}}{(2 \pi)^{2}} \frac{\vec{p} \cdot \vec{k}}{\vec{p}^{2}} \frac{A(\vec{k})}{\omega^{2}+v_{F}^{2} A^{2}(\vec{k}) \vec{k}^{2}+\Delta^{2}(\vec{k})} D(\omega, \vec{p}-\vec{k}), \\
& \Delta(\vec{p})=\frac{e^{2}}{\varepsilon} \int_{-\infty}^{\infty} \frac{d \omega}{2 \pi} \int \frac{d^{2} \vec{k}}{(2 \pi)^{2}} \frac{\Delta(\vec{k})}{\omega^{2}+v_{F}^{2} A^{2}(\vec{k}) \vec{k}^{2}+\Delta^{2}(\vec{k})} D(\omega, \vec{p}-\vec{k}) .
\end{aligned}
$$

In order to evaluate these equations at the critical coupling we apply bifurcation theory [29], which amounts to linearizing Eqs. $(2.6,2.7)$ around the point at which the nontrivial solution for the mass function bifurcates away from the trivial one. In this case the integral equation for $A$ decouples. Furthermore employing a GW approximation as in Ref. [30] amounts to setting $A=1$ on the r.h.s. of Eq. (2.6) such that the corresponding equation is reduced to solving a single integral. The integration over the (Euclidean) frequency $\omega$ can then be performed analytically [10], yielding (now with $p \equiv|\vec{p}|$ ):

$$
A(p)=1+\frac{e^{2}}{v_{F} \varepsilon} \frac{1}{\vec{p}^{2}} \int \frac{d^{2} \vec{k}}{(2 \pi)^{2}} \frac{\vec{p} \cdot \vec{k}}{k|\vec{p}-\vec{k}|} J(z, g),
$$

where $g=e^{2} N_{f} \pi /\left(4 \varepsilon v_{f}\right)$ and the piecewise function $J(z, g)$ with $z \equiv|\vec{k}| /|\vec{p}-\vec{k}|$ is given by

$$
J(z, g)=\frac{\left(z^{2}-1\right)[\pi-g c(z)]+z g^{2} c(g)}{z^{2}+g^{2}-1}, \text { with } c(x)=\left\{\begin{array}{ll}
2 \operatorname{arccosh}(x) / \sqrt{x^{2}-1}, & x>1 \\
2 \arccos (x) / \sqrt{1-x^{2}} & , x<1 \\
2 & , x=1
\end{array} .\right.
$$

At this point, it is common to further approximate the rather complicated integration over the angle between $\vec{p}$ and $\vec{k}$ [10]. Here we proceed differently using $z$ as an integration variable to encode the 
dependence on this angle. Then the standard angular and radial integrals are replaced by

$$
\int_{0}^{\frac{\Lambda}{p}} d \beta \int_{\frac{\beta}{1+\beta}}^{\frac{\beta}{|1-\beta|}} d z=\int_{0}^{1} d z \int_{\frac{z}{z+1}}^{\frac{z}{1-z}} d \beta+\int_{1}^{\frac{\Lambda}{p}} d z \int_{\frac{z}{z+1}}^{\frac{z}{z-1}} d \beta,
$$

where $\beta=k / p$. With this trick, the integral over $z$ in Eq. (2.8) nicely splits into intervals that correspond to the definition domains of the function $J$ in Eq. (2.9). A straightforward calculation then leads to the following complete result for the Fermi velocity dressing function in the GW approximation with RPA polarization effects in the Coulomb interaction:

$$
A(p)=1+\frac{2}{\pi^{2} N_{f} g}\left\{-\left[\pi-2 g+c(g)\left(g^{2}-1\right)\right] \ln \frac{p}{\Lambda}+f(g)+(\pi+4 g) \ln 2-4 g\right\},
$$

where the physical cutoff $\Lambda$ is determined by the size of the Brillouin zone. The logarithmic term comes from the leading one-loop correction and was obtained in Ref. [10] already. Here we include all nonsingular terms which were not given therein. They can be expressed in terms of the function

$$
\begin{aligned}
f(g)= & -2\left(g^{2}-1\right)\left\{g \sum_{n=1}^{\infty} \frac{\left(g^{2}-1\right)^{n-1} v_{n}-\delta_{n}}{g^{2 n}}+\frac{1}{\sqrt{g^{2}-1}}\left[\operatorname{arccosh} g \ln \left(2 \frac{\sqrt{g^{2}-1}}{g^{2}}\right)\right.\right. \\
& \left.\left.-\pi \arctan \frac{\sqrt{g^{2}-1}-1}{\sqrt{g^{2}-1}+1}\right]\right\} .
\end{aligned}
$$

The sum in first term on the right, involving the coefficients

$$
\delta_{n}=\int_{0}^{1} d z\left(1-z^{2}\right)^{n-3 / 2} \arccos z \quad \text { and } v_{n}=\int_{0}^{1} d t t\left(1-t^{2}\right)^{n-3 / 2} \arccos \frac{1}{t}
$$

can be evaluated numerically. The full GWA result (2.11) for the Fermi velocity renormalization function at various values of the bare effective coupling $\alpha=e^{2} / \varepsilon v_{f}$ is shown in Fig. 1. The logarithmic singularity at $p^{2} \rightarrow 0$ is rather weak at weak coupling and the nonsingular part of the radiative corrections remains small as well. This changes when $\alpha \approx 2$ is used for suspended graphene. Both logarithmic and regular contributions are considerably larger and a correspondingly strong renormalization of the Fermi velocity results. If we simply use $v_{f} A\left(\mu / v_{f}\right)$ to estimate the renormalized Fermi velocity at nonzero chemical potential $\mu$ for a finite charge-carrier density this result describes the recent suspended-graphene experiments [22] so well that a more quantitative comparison maybe worthwhile [28]. The large effect also shows that a nontrivial dressing function $A(p)$ should be included in the analysis of the semimetal-insulator transition at strong coupling, instead of using $A=1$ as in [10]. We will turn to this analysis next.

Having calculated the function $A$ in GWA, we now use this result in the bifurcation analysis of the gap equation around the critical point. At leading order in $\Delta$ the gap equation then reduces to:

$$
\Delta(p)=\frac{e^{2}}{v_{F} \varepsilon} \int \frac{d^{2} \vec{k}}{(2 \pi)^{2}} \frac{1}{k|\vec{p}-\vec{k}|} \frac{\Delta(k)}{A(k)} J(z A(k), g) .
$$

We proceed by inserting the Fermi velocity dressing function Eq. (2.11) into the above equation, and solve the resulting equation by using standard numerical methods. Note that in this case, not only the logarithmic but also the regular contributions to $A$ become important at strong coupling. 


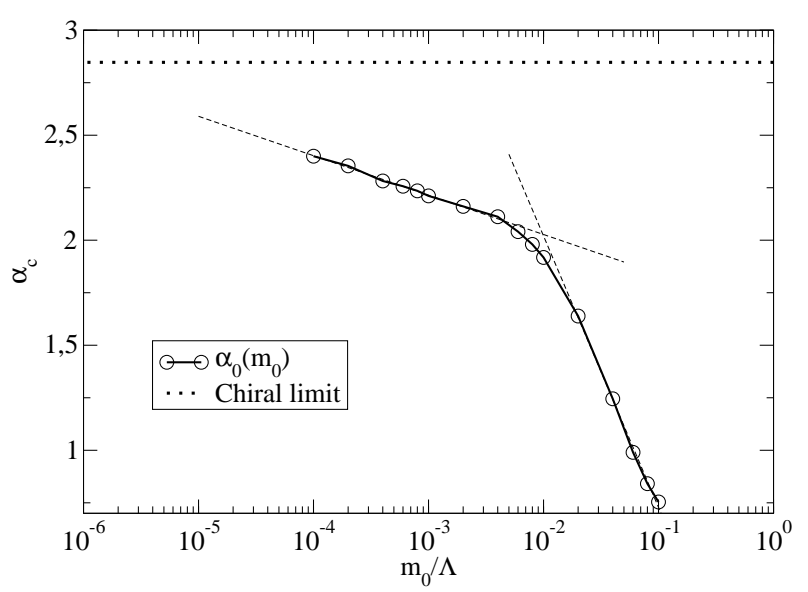

Figure 2: Mass dependence of the critical coupling for $N_{f}=2$.

For the critical coupling we then obtain $\alpha_{c}=2.85$ which is larger than the bare coupling $\alpha_{0}=2.19$ with $\varepsilon=1$ for suspended graphene. This suggests that the Coulomb interactions might not be strong enough to generate a gapped phase in the electronic spectrum of suspended graphene. Our result is thus in contrast to the many previous studies mentioned in the introduction. The values predicted there all lie below the bare coupling for suspended graphene. In particular, our results are directly comparable to the work of Gamayun et al. in Ref. [10] in which the same gap equation with the same RPA improved Coulomb interaction was solved, however, with $A=1$, i.e., with neglecting Fermi velocity renormalization effects, leading to $\alpha_{c}=0.92$.

As it turns out, lattice simulations at present also appear to underestimate the value of the critical coupling $[17,20]$. Hence, in order to mimic the lattice setup, where a non-zero fermion mass term must be used in a finite volume, ${ }^{1}$ we have introduced such an explicitly symmetry breaking term as a seed in the gap equation (2.14) here as well, and calculated the corresponding pseudocritical couplings. Analyzing the results displayed in Fig. 2, we notice that the diagram contains two distinct regions, each of them displaying a logarithmic dependence of the critical coupling on the bare mass (dashed lines in the figure). The scale separating the two regions, where the dynamically generated mass is bigger or smaller than the bare fermion mass, is of the order of magnitude $m_{0} / \Lambda=10^{-2}$. Note however that a proper comparison with the lattice requires taking into account the different UV scales, as graphene lattice simulations have been performed on both hexagonal [20] and square [17] lattices. Further studies will be necessary to clarify the issue.

\section{Summary and outlook}

In this work, we have provided an estimate of the critical coupling for the semimetal-insulator phase transition in graphene. We have employed the Dyson-Schwinger equation for the fermion

\footnotetext{
${ }^{1}$ Finite-volume effects must also be studied carefully on the lattice. Here $\mathrm{QED}_{3}$ serves as an important example where it has been shown what a significant impact these can have, see for example [31] and the references therein.
} 
propagator in the Dirac cone approximation with a bare vertex and Coulomb interactions including frequency dependent polarization effects at the RPA level. Importantly, however, we have explicitly taken into account the GWA running of the Fermi velocity. In this setting, we have obtained a critical coupling of $\alpha_{c}=2.85$, which is larger than the bare coupling $\alpha_{0}=2.19$ for suspended graphene. This allows us to conclude that the logarithmic growth of the Fermi velocity near charge neutrality weakens the Coulomb interaction preventing the emergence of a gapped phase, in agreement with the experimental observations [22].

Despite the qualitative agreement with experimental observations, a complete understanding of the physical picture calls for a full nonperturbative investigation, i.e., to solve the coupled system of integral equations for the photon and fermion propagators simultaneously. It is likely that including the nonperturbative gauge propagator and improving the vertex functions may lead to further corrections of the critical coupling. We recall that nonperturbative contributions to both the photon propagator and vertex functions lead to significant corrections in ordinary $\mathrm{QED}_{3}$, of the order of twenty percent for the photon propagator and ten percent for the vertex [27, 32]. These effects, along with finite volume and mass corrections, might in the end together explain the discrepancy between experiment and lattice simulations [17,20].

Moreover, induced four-fermion contact interactions might become important at strong coupling as well. Such local four-fermion interaction terms as allowed by the symmetries should be considered, e.g., to model electron-phonon interactions, in addition to the long-range Coulomb fields in the effective low-energy continuum theory for the electronic excitations of graphene in the future. ${ }^{2}$ Finally, another interesting problem is the inclusion a chemical potential for a finite density of charge carriers as induced experimentally, e.g., by chemical doping. While the fermionsign problem makes this problem hard to address with lattice simulations, the modifications to the Dyson-Schwinger equations are quite straightforward. The resulting effects of a finite chargecarrier density on the running Fermi velocity are the subject of an upcoming publication.

\section{Acknowledgments}

We would like to thank P. Buividovich and M. Polikarpov for valuable discussions. This work was supported by the Deutsche Forschungsgemeinschaft within the SFB 634, the Helmholtz International Center for FAIR within the LOEWE program of the State of Hesse, and the Helmholtz Young Investigator Group No. VH-NG-332.

\section{References}

[1] K. S. Novoselov, A. K. Geim, S. V. Morozov, D. Jiang, Y. Zhang, S. V. Dubonos, I. V. Grigorieva, and A. A. Firsov, Science 306, 666 (2004), arXiv : cond-mat/ 0410550.

[2] V. N. Kotov, B. Uchoa, and A. H. Castro Neto, Phys. Rev. B 78, 035119 (2008), arXiv:0706.2185.

[3] A. Castro Neto, F. Guinea, N. Peres, K. Novoselov, and A. Geim, Rev. Mod. Phys. 81, 109 (2009).

[4] V. N. Kotov, B. Uchoa, V. M. Pereira, F. Guinea, and A. H. Castro Neto, Rev. Mod. Phys. 84, 1067 (2012).

\footnotetext{
${ }^{2}$ For first studies in this direction we refer the reader to Refs. [10, 13].
} 
[5] J. González, F. Guinea, and M. A. H. Vozmediano, Nucl. Phys. B 424, 595 (1994), arXiv:hep-th/9311105.

[6] J. González, F. Guinea, and M. A. Vozmediano, Phys. Rev. B 63, 134421 (2001), arXiv:cond-mat/0007337.

[7] F. de Juan, A. G. Grushin, and M. A. H. Vozmediano, Phys. Rev. B 82, 125409 (2010), arXiv: 1002.3111.

[8] E. V. Gorbar, V. P. Gusynin, and V. A. Miransky, Phys. Rev. D 64, 105028 (2001), arXiv:hep-ph/0105059.

[9] E. V. Gorbar, V. P. Gusynin, V. A. Miransky, and I. A. Shovkovy, Phys. Rev. B 66, 045108 (2002), arXiv:cond-mat/0202422.

[10] O. V. Gamayun, E. V. Gorbar, and V. P. Gusynin, Phys. Rev. B 81, 075429 (2010), arXiv:0911.4878.

[11] D. V. Khveshchenko, Phys. Rev. Lett. 87, 246802 (2001), arXiv : cond-mat/ 0101306.

[12] D. V. Khveshchenko, J. Phys. Cond. Mat. 21, 075303 (2009), arXiv: 0807 . 0676.

[13] D. Mesterhazy, J. Berges, and L. von Smekal, Phys. Rev. B 86, 245431 (2012), arXiv : 1207.4054.

[14] J. E. Drut and D. T. Son, Phys. Rev. B 77, 075115 (2008), arXiv: 0710.1315.

[15] I. F. Herbut, Phys. Rev. Lett. 97, 146401 (2006), arXiv: cond-mat/ 0606195.

[16] J. E. Drut and T. A. Lähde, Phys. Rev. Lett. 102, 026802 (2009a), arXiv : 0807 . 0834.

[17] J. E. Drut and T. A. Lähde, Phys. Rev. B 79, 165425 (2009b), arXiv: 0901.0584.

[18] J. E. Drut and T. A. Lahde, Phys. Rev. B 79, 241405 (2009), arXiv: 0905.1320.

[19] P. Buividovich and M. Polikarpov (2012), arXiv:1206.0619.

[20] P. Buividovich, E. Luschevskaya, O. Pavlovsky, M. Polikarpov, and M. Ulybyshev, Phys. Rev. B 86, 045107 (2012), arXiv: 1204.0921.

[21] O. Vafek and M. J. Case, Phys. Rev. B 77, 033410 (2008), arXiv: 0710.2907.

[22] D. C. Elias, R. V. Gorbachev, A. S. Mayorov, S. V. Morozov, A. A. Zhukov, P. Blake, L. A. Ponomarenko, I. V. Grigorieva, K. S. Novoselov, F. Guinea, et al., Nature Physics 8, 172 (2012).

[23] R. Alkofer and L. von Smekal, Phys. Rept. 353, 281 (2001), arXiv: hep-ph/ 0007355.

[24] P. Maris and C. D. Roberts, Int. J. Mod. Phys. E 12, 297 (2003), arXiv: nucl-th/ 0301049.

[25] C. S. Fischer, J. Phys. G 32, R253 (2006), arXiv: hep-ph/ 0605173.

[26] P. Maris, Phys. Rev. D 52, 6087 (1995), arXiv: hep-ph/ 9508323.

[27] C. S. Fischer, R. Alkofer, T. Dahm, and P. Maris, Phys. Rev. D 70, 073007 (2004), arXiv:hep-ph/0407104.

[28] C. Popovici, C. S. Fischer, and L. von Smekal, in preparation.

[29] D. Atkinson, J. C. R. Bloch, V. P. Gusynin, M. R. Pennington, and M. Reenders, Phys. Lett. B 329, 117 (1994).

[30] J. González, F. Guinea, and M. A. Vozmediano, Phys. Rev. B 59, 2474(R) (1999), arXiv:cond-mat/9807130.

[31] T. Goecke, C. S. Fischer, and R. Williams, Phys. Rev. B 79, 064513 (2009), arXiv: 0811.1887.

[32] J. Braun, C. S. Fischer, and H. Gies, Phys. Rev. D 84, 034045 (2011), arXiv: 1012.4279. 